\title{
Relationship of polymorphisms in the cholesteryl ester transport protein gene R451Q with coronary heart disease and diabetes in Uyghur and Han Chinese
}

\author{
C.J. Zhang1, H.M. Qiu² and J.P. Qiu' \\ ${ }^{1}$ Department of Cardiology, Gongli Hospital, District Pudong, Shanghai, China \\ ${ }^{2}$ Cadre Ward, First Affiliated Hospital, Xinjiang Medical University, Xinjiang, China \\ Corresponding author: C.J. Zhang \\ E-mail: chenjunzhangcn@163.com
}

Genet. Mol. Res. 13 (1): 954-962 (2014)

Received December 13, 2012

Accepted June 17, 2013

Published February 19, 2014

DOI http://dx.doi.org/10.4238/2014.February.19.6

\begin{abstract}
To investigate the relationship of polymorphisms in the cholesteryl ester transport protein (CETP) gene with coronary heart disease (CHD) and diabetes in subjects of Uyghur and Han Chinese origin, 266 subjects with CHD including 154 subjects with type 2 diabetes mellitus and 136 healthy subjects (as a control group) were enrolled in this study. Polymerase chain reaction and an enzymatic assay based on the ligase detection reaction were used to detect R451Q polymorphisms in the CETP gene. The data were used for genotyping to determine the allele frequency distribution of the CETP gene R451Q polymorphism to investigate its effects on lipid and apolipoprotein levels. Genotype and allele frequencies of CETP R451QA did not show any significant differences among the CHD and healthy control groups. Moreover, no significant difference in the CETP R451QA genotype and allele frequency was detected among the subjects of Uyghur and Han origin. Blood levels of lipids and apolipoproteins likewise lacked an association with CETP R451QA
\end{abstract}


genotype frequencies in the $\mathrm{CHD} /$ diabetes group. We conclude that the R451Q polymorphisms in the CETP gene had no effects on blood lipid levels and are not a risk factor for CHD in Han and Uyghur Chinese.

Key words: CETP R451Q; Coronary heart disease with diabetes; Polymorphism

\section{INTRODUCTION}

Cholesteryl ester transfer protein (CETP) is one of the most important determinants of plasma high-density lipoprotein cholesterol (HDL-C) levels. A large amount of epidemiological data indicate that CETP levels in plasma is related to HDL-C, low-density lipoprotein cholesterol (LDL-C), apolipoprotein A1 (ApoA1), and other lipids and lipoproteins, and that CETP may be involved in the regulation of lipid-particle size (Liu et al., 2002; Lu et al., 2003; Barter et al., 2007; Papp et al., 2012). This relationship may be affected by certain lifestyles and physiological factors, including drinking, smoking, blood pressure, body mass index, and physical exercise. Studies with transgenic mice expressing CETP at different levels have shown that the levels of HDL-C, chyle microparticles, very LDL (VLDL), intermediate-density lipoprotein (IDL), and LDL in plasma changed with changes in CETP concentration. CETP in plasma mostly binds to HDL, and the components containing ApoA1 lipoprotein are closely associated with the occurrence and development of atherosclerosis.

CETP is a hydrophobic sugar protein composed of 476 amino acids. It mainly mediates reverse transport of cholesterol, transporting excess cholesterol in cells of the peripheral tissue to the liver via HDL for metabolization. VLDL, IDL, and LDL are transported, and IDL and LDL are degraded by LDL receptors in the liver, whereas triglycerides (TG) are transported in the opposite direction at an equal molecular ratio. Thus, HDLs become rich in TG and are hydrolyzed by hepatic TG lipase. The HDL particles may be recycled, thereby accelerating cholesterol efflux from peripheral tissues and maintaining stability of intracellular cholesterol levels. The expression of the CETP gene plays a decisive role in the transfer of lipids and lipoproteins in vivo. Artificial alteration of CETP activity causes changes in lipid metabolism. For example, intravenous injection of CETP monoclonal or polyclonal antibodies results in changes of lipoproteins that include increased levels of HDL-C and decreased levels of VLDL-C and LDL-C.

Studies have shown that the CETP gene determines levels of HDL-C, and that it is an independent risk factor for AS and cardiovascular disease (Park et al., 2003; Spielmann et al., 2007; McCaskie et al., 2007; Kathiresan et al., 2009; Papp et al., 2012; Khovidhunkit et al., 2012). The relationships of gene polymorphisms in CETP with coronary heart disease (CHD), blood lipids, and lipoproteins and apolipoproteins have been extensively studied, but were found to be inconsistent (Boekholdt and Thompson, 2003). Studies of polymorphisms in the CETP gene have mainly focused on the Japanese population, in which the rate of CETP gene mutations is high (up to 7\% in certain sites) and gene mutations involving multiple loci and having familial associations may exist.

Studies of CETP gene polymorphisms in other populations, such as Australian immigrants and Jewish groups, and from North America, Europe, and South Korea have also been 
reported (Inazu et al., 1994; Yamashita et al., 2000; Ordovas, 2000; Ordovas et al., 2000), but CETP mutations were identified in Caucasian Europeans and Americans. It is suggested that the CETP gene in people of different race has different mutations. The first place of the 14th intron of CETP occurred in GA mutant lines, mutation frequency of R451Q is low; however, the activity of the mutant CETP protein is often very high, and, therefore, it has a certain value for research.

The incidence of CHD and diabetes is high in the Xinjiang Uyghur population, but the CETP gene R451Q polymorphism has not been reported from this population, and the relationship of the CETP R451Q polymorphism with CHD is not clear. Therefore, to determine the relationship of the CETP gene polymorphism with CHD and diabetes in subjects of Uyghur or Han origin, this study was designed to investigate the distribution of the R451Q polymorphism of the CETP gene in these groups; its relationship with CHD and diabetes; and its effect on blood lipids, lipoprotein, and apolipoprotein levels, to provide a scientific basis for early diagnosis and prevention of these diseases.

\section{MATERIAL AND METHODS}

\section{Study subjects}

From January to September 2008, 266 inpatients in the heart center of the First Affiliated Hospital of Xinjiang Medical Center were selected. These subjects included 196 males and 70 females who had a clear history of acute myocardial infarction or whose angiographies indicated $\geq 50 \%$ stenosis of at least one main artery and who had a history of typical angina pectoris. Of these subjects, 154 (110 males and 44 females) also had diabetes mellitus. All of the subjects were not treated with any lipid-lowering drugs within 2 weeks before the examination, and the following subjects were excluded: subjects with cerebrovascular accidents or those with peripheral vascular disease, tuberculosis, malignant tumors, blood diseases, autoimmune diseases, thyroid disease, chronic liver disease or kidney disease, or with a history of these diseases. Diabetes in the selected inpatients was diagnosed according to the International Diabetes Asia Pacific diagnostic criteria.

In total, 136 healthy volunteers (76 males and 60 females), identified by an examination in the Examination Center of the First Affiliated Hospital of Xinjiang Medical and inpatients who were diagnosed as free from stenosis in coronary angiography during the same study period, were selected as the control group. To qualify as healthy, absence of the following conditions was required: hypertension, diabetes, CHD, cerebrovascular accident, peripheral vascular disease, tuberculosis, malignant tumors, blood diseases, autoimmune diseases, thyroid disease, chronic liver disease or kidney disease history, or an electrocardiogram (ECG) indicating an abnormal condition.

\section{Methods}

General information about the inpatients was collected through questionnaires and past medical history. A routine physical examination, 12-lead ECG, and blood biochemical parameters of the selected inpatients were conducted. 


\section{Collection of blood specimen}

After $12 \mathrm{~h}$ of fasting, on the next morning, the height, weight, and blood pressure of the inpatients were measured and the body mass index (BMI = body weight $[\mathrm{kg}] /$ height $\left[\mathrm{m}^{2}\right]$ ) was calculated. In total, $3 \mathrm{~mL}$ blood was collected and serum was obtained through centrifugation. A routine biochemical examination was performed and another $3 \mathrm{~mL}$ blood was collected and stored at $-80^{\circ} \mathrm{C}$ for DNA extraction.

\section{Routine determination of blood biochemical parameters}

The serum total cholesterol (TC), TG, HDL-C, LDL-C, ApoA1, ApoB, and fasting blood glucose (FBG) were detected and measured by clinical laboratory professionals.

\section{DNA extraction and genotype analysis}

A genomic DNA extraction kit (Shanghai Sangon Limited Company) was used to extract genomic DNA.

\section{Primer design}

According to the sequence of the CETP gene given in the literature (Kakko et al., 1998; Maruyama et al., 2003), the following primers were designed: upstream primer (5'-AGCCCT CATGAACAGCAAAG-3') and downstream primer (5'-CCTCTGTCTGTCTCCCCAAC-3'). PCR amplifications were performed in a Perkin Elmer Gene Amp PCR Systems 9600 with the following thermal cycling protocol: $95^{\circ} \mathrm{C}$ denaturation for $10 \mathrm{~min}$, followed by 35 cycles of $95^{\circ} \mathrm{C}$ for $30 \mathrm{~s}, 60^{\circ} \mathrm{C}$ for $50 \mathrm{~s}$, and $72^{\circ} \mathrm{C}$ for $60 \mathrm{~s}$; a final extension step was performed at $72^{\circ} \mathrm{C}$ for $10 \mathrm{~min}$. The PCR products were analyzed by an enzymatic method based on NEB's Taq DNA ligase enzyme system and supporting software. It included $100 \mu \mathrm{L} 10 \mathrm{X}$ buffer, $100 \mu \mathrm{L}$ of the Probe MIX containing $5 \mathrm{U}$ ligase, and $695 \mu \mathrm{L}$ deionized water. After mixing and centrifugation, $1 \mu \mathrm{L}$ PCR product was added to $9 \mu \mathrm{L}$ ligase enzyme mix.

Reactions were performed in a Perkin Elmer Gene Amp PCR Systems 9600, and consisted of 1 cycle of $95^{\circ} \mathrm{C}$ for $2 \mathrm{~min}$, followed by 35 cycles of $94^{\circ} \mathrm{C}$ for $30 \mathrm{~s}$ and $60^{\circ} \mathrm{C}$ for $2 \mathrm{~min}$. The LDR connection product $(1 \mu \mathrm{L})$ was mixed with $1 \mu \mathrm{LABI}$ GS-500 ROX fluorescent-labeled molecular weight standards and $1 \mu \mathrm{L}$ to sample liquid in ion carboxamide. The samples were heat denatured at $95^{\circ} \mathrm{C}$ for $2 \mathrm{~min}$, quenched on ice, and subjected to electrophoresis for $2.5 \mathrm{~h}$ in a 5\% polyacrylamide and $5 \mathrm{M}$ urea at $3000 \mathrm{~V}$. Data were collected with the GENESCANTM672 software and the GeneMapper software was used for data analysis and genotyping.

\section{Statistical analysis}

The SPSS13.0 software package was used for statistical analysis. The gene and genotype frequencies of the CETP R451Q polymorphism in the CHD and control groups were determined by gene notation, and the chi-square test was used to determine the genotype and allele frequencies and to test for Hardy-Weinberg equilibrium. The chi-square test was also used to compare the genotype distribution and allele frequency in the CHD group, CHD with 
diabetes group, and the control group. For the measurement data, $t$-test, single-factor variance analysis, or the multiple-rank sum test for independent samples was used depending on whether data had a normal distribution and had homogeneous variances. A bilateral probability of $\mathrm{P}$ $<0.05$ was considered as statistically significant.

\section{PCR product of the ligase detection reaction}

In total, 377 sequences were analyzed by electrophoresis. Probe: Probe name sequence (5'-3') LDR length RS1800777_MODIFY P-GAGTGATAATCTCAGGGTTGATGATTTTTT TTTTTTTTTTTTTTT-FAM RS1800777_ATTTTTTTTTTTTTTTTTTTTGAGGGGGGC TTTGTACTCACATCTT 90 RS1800777_GTTTTTTTTTTTTTTTTTTTTTTGAGGGGGG CTTTGTACTCACATCTC 92 (Figure 1).

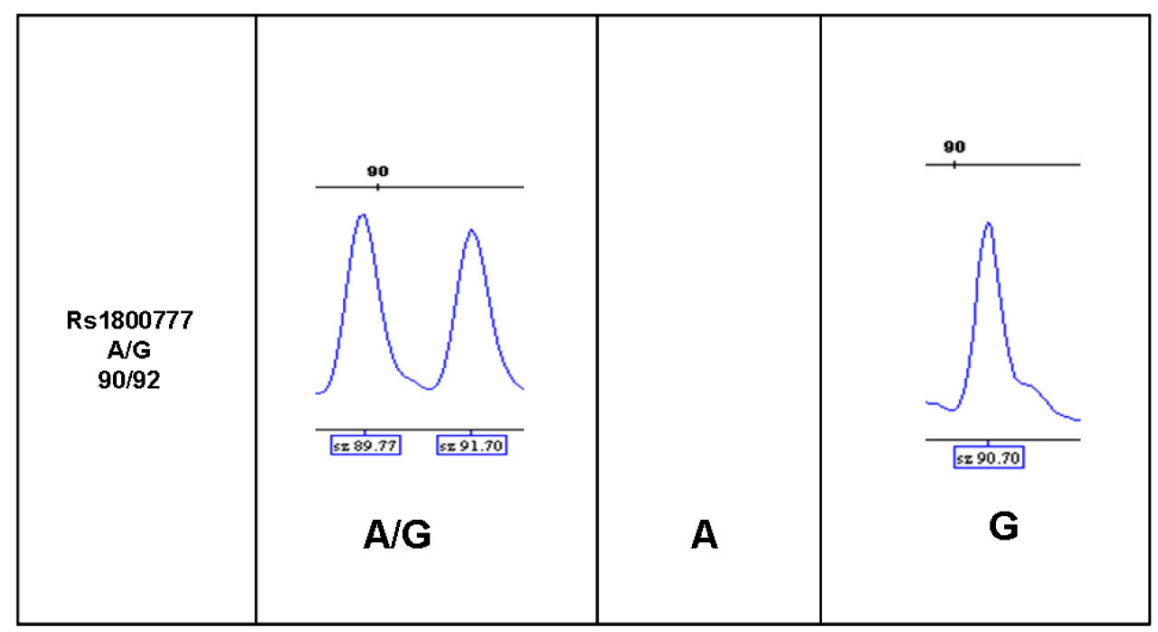

Figure 1. PCR product of the ligase detection reaction.

\section{RESULTS}

\section{Clinical data and biochemical indicators in the two groups}

The mean age of the patients in the two groups was higher than that of the control group (CHD with diabetes group, LDL-C rank sum test).

The BMI, TG, LDL-C, ApoB/A, and LP-a of the subjects in the CHD group were significantly higher than those of the subjects in the control group $(\mathrm{P}<0.05)$. On the other hand, the ApoA1 of the patients on CHD group was significantly lower than that of the patients in the control group $(\mathrm{P}<0.05)$.

The BMI, FBG, TC, TG, ApoB and ApoB/A, and LP-a of the subjects in the CHD combined with diabetes group were higher than those of the subjects in the control group $(\mathrm{P}<$ 0.05), whereas HDL-C of the subjects in the CHD combined with diabetes group was lower than that of the subjects in the control group $(\mathrm{P}<0.05)$ (Table 1). 


\begin{tabular}{|c|c|c|c|}
\hline & Control & CHD & CHD combined with diatetes \\
\hline Cases (N) & 136 & 112 & 154 \\
\hline Age (years) & $47.48 \pm 11.07$ & $59.40 \pm 10.92 *$ & $62.22 \pm 9.09 *$ \\
\hline BMI $\left(\mathrm{kg} / \mathrm{m}^{2}\right)$ & $24.86 \pm 3.91$ & $26.38 \pm 4.19^{*}$ & $26.64 \pm 3.76^{*}$ \\
\hline FBG (mM) & $5.28 \pm 1.24$ & $5.23 \pm 0.81$ & $7.81 \pm 2.88^{*}$ \\
\hline $\mathrm{TC}(\mathrm{mM})$ & $3.02 \pm 0.77$ & $3.22 \pm 0.95$ & $3.45 \pm 1.38^{*}$ \\
\hline $\mathrm{TG}(\mathrm{mM})$ & $1.69 \pm 0.88$ & $1.94 \pm 1.02 *$ & $2.24 \pm 1.70^{*}$ \\
\hline HDL-C (mM) & $1.14 \pm 0.29$ & $1.08 \pm 0.42$ & $1.02 \pm 0.26^{*}$ \\
\hline LDL-C (mM) & $2.36 \pm 0.78$ & $2.75 \pm 0.90 *$ & $2.68 \pm 0.80^{\triangle}$ \\
\hline ApoA (g/L) & $1.36 \pm 0.16$ & $1.24 \pm 0.16^{*}$ & $1.31 \pm 0.23$ \\
\hline ApoB $(g / L)$ & $0.84 \pm 0.20$ & $0.84 \pm 0.22$ & $0.91 \pm 0.22 *$ \\
\hline $\mathrm{ApoB} / \mathrm{A}(\mathrm{g} / \mathrm{L})$ & $0.62 \pm 0.17$ & $0.68 \pm 0.17^{*}$ & $0.71 \pm 0.19^{*}$ \\
\hline $\mathrm{LP}-\mathrm{a}(\mathrm{mM})$ & $101.54 \pm 66.88$ & $152.38 \pm 67.69^{*}$ & $169.14 \pm 87.11^{* \triangle}$ \\
\hline
\end{tabular}

Data are reported as means $\pm \mathrm{SD} . * \mathrm{P}<0.05, \triangle$ heterogeneity of variance, rank sum test.

The Hardy-Weinberg test indicated that the genotype distribution of the CETP R451Q locus was consistent with the law of genetic equilibrium and consistent with the distribution of the allele frequencies in different ethnic populations.

\section{Genotype frequencies and allele frequency distribution of the CETP R451Q polymorphism}

The homozygous AA genotype for the CETP R451Q polymorphism was not detected in the two populations in this study. The mutation frequency of the CETP gene R451Q in the CHD group was $4.5 \%(5 / 112)$, that of the CHD combined with diabetes group was $3.9 \%$ $(6 / 154)$, and that of the control group was $3.7 \%(5 / 136)$. The allele frequency calculations did not meet the conditions of the chi-square test. Results of the Fisher test indicated that the mutation frequencies in the CHD groups and the control group were not statistically significant (P $>0.05$ ). The allele frequency was $2.2,2.0$, and $1.8 \%$. Although the A allele frequency of the subjects in the CHD group was higher than that in the control group, this difference was not statistically significant (Table 2).

\begin{tabular}{|c|c|c|c|c|c|c|}
\hline \multirow[t]{2}{*}{ Group cases } & \multicolumn{2}{|c|}{ Genotype frequencies [N (\%)] } & \multirow[t]{2}{*}{$\mathrm{P}$} & \multicolumn{2}{|c|}{ Allele } & \multirow[t]{2}{*}{$\mathrm{P}$} \\
\hline & GG & $\overline{\mathrm{AG}}$ & & G & A & \\
\hline Control $(\mathrm{N}=136)$ & $131(96.3)$ & $5(3.7)$ & & $266(98.2)$ & $5(1.8)$ & \\
\hline $\mathrm{CHD}(\mathrm{N}=112)$ & $107(95.5)$ & $5(4.5)$ & 0.198 & $221(97.8)$ & $5(2.2)$ & 0.522 \\
\hline $\mathrm{CHD}+\mathrm{DM}(\mathrm{N}=154)$ & $148(96.1)$ & $6(3.9)$ & 0.519 & $305(98.0)$ & $6(2.0)$ & 0.202 \\
\hline
\end{tabular}

\section{Blood lipid levels in the subjects from the CHD group with different CETP gene R451Q genotypes}

No statistically significant differences in the levels of blood lipids and blood glucose were detected among subjects from of the CHD group with the GG and GA genotypes (Table 3). 
Table 3. Comparison of blood lipid level of the patients in the CHD group with different CETP gene genotypes.

\begin{tabular}{|c|c|c|c|}
\hline \multirow[t]{2}{*}{ CHD group } & \multicolumn{2}{|c|}{ Genotype } & \multirow[t]{2}{*}{$P$} \\
\hline & GG & AG & \\
\hline Cases & 107 & 5 & - \\
\hline $\mathrm{TC}(\mathrm{mM})$ & $4.11 \pm 0.84$ & $3.93 \pm 1.18$ & 0.510 \\
\hline $\mathrm{TG}(\mathrm{mM})$ & $2.07 \pm 1.06$ & $2.02 \pm 1.22$ & 0.898 \\
\hline HDL-C (mM) & $1.12 \pm 0.36$ & $1.02 \pm 0.32$ & 0.421 \\
\hline LDL-C (mM) & $2.54 \pm 0.85$ & $2.55 \pm 1.10$ & 0.975 \\
\hline BMI $\left(\mathrm{kg} / \mathrm{m}^{2}\right)$ & $25.45 \pm 4.03$ & $27.81 \pm 5.33$ & 0.076 \\
\hline Lp-a (mM) & $185.87 \pm 155.02$ & $289.06 \pm 325.20$ & $0.811^{\triangle}$ \\
\hline Blood glucose (mM) & $5.25 \pm 1.04$ & $5.16 \pm 0.75$ & 0.786 \\
\hline
\end{tabular}

\section{Blood lipid levels in the subjects from the CHD combined with diabetes group with different CETP gene R451Q genotypes}

No statistically significant differences in the levels of blood lipids and blood glucose were detected among subjects from of the CHD group combined with diabetes group with the GG and GA genotypes (Table 4).

\begin{tabular}{|c|c|c|c|}
\hline \multirow[t]{2}{*}{ CHD combined with diabetes } & \multicolumn{2}{|c|}{ Genotype } & \multirow[t]{2}{*}{$P$} \\
\hline & GG & AG & \\
\hline Cases & 149 & 6 & - \\
\hline TC (mM) & $4.26 \pm 1.19$ & $3.82 \pm 1.08$ & 0.224 \\
\hline $\mathrm{TG}(\mathrm{mM})$ & $2.12 \pm 1.42$ & $2.34 \pm 1.61$ & 0.616 \\
\hline HDL-C (mM) & $1.08 \pm 0.29$ & $1.03 \pm 0.31$ & 0.562 \\
\hline LDL-C (mM) & $2.41 \pm 0.91$ & $2.15 \pm 0.88$ & 0.347 \\
\hline BMI $\left(\mathrm{kg} / \mathrm{m}^{2}\right)$ & $25.79 \pm 3.91$ & $26.89 \pm 3.93$ & 0.363 \\
\hline Lp-a (mM) & $199.26 \pm 162.24$ & $243.82 \pm 191.01$ & 0.376 \\
\hline Blood glucose $(\mathrm{mM})$ & $6.60 \pm 2.56$ & $5.90 \pm 1.40$ & 0.363 \\
\hline
\end{tabular}

\section{Comparisons of genotype and allele frequencies in Uyghur and Han subjects}

The Uyghur subjects had frequencies of the AG genotype and $G$ allele that were higher than in the Han subjects, but this difference was not statistically significant. There was no significant difference in genotype and allele frequencies between the control group and the CHD group (including the CHD with diabetes group) when subjects were matched according to ethnic origin.

When the two different ethnic groups were compared, the genotype and allele frequencies of the Uyghur subjects were higher than those in the Han subjects, but this difference was again not statistically significant (Tables 5 and 6).

Table 5. Comparison of genotype and allele frequencies of the patients with CHD in Uighur and Han.

\begin{tabular}{|c|c|c|c|c|}
\hline & \multicolumn{2}{|c|}{ Genotypic frequency } & \multicolumn{2}{|c|}{ Allele frequency } \\
\hline & GG & AG & G & A \\
\hline Han & $57(96.6 \%)$ & $2(3.4 \%)$ & $116(98.3 \%)$ & $2(1.7 \%)$ \\
\hline Uighur & $50(94.3 \%)$ & $3(5.7 \%)$ & $103(97.1 \%)$ & $3(2.9 \%)$ \\
\hline Fisher & & & & \\
\hline $\mathrm{P}$ & & & & \\
\hline
\end{tabular}


Table 6. Comparison of genotype and allele frequencies of the patients with CHD combined with diabetes in Uighur and Han.

\begin{tabular}{|c|c|c|c|c|}
\hline & \multicolumn{2}{|c|}{ Genotypic frequency } & \multicolumn{2}{|c|}{ Gene frequency } \\
\hline & GG & $\mathrm{AG}$ & G & $\mathrm{A}$ \\
\hline Han & $86(97.7 \%)$ & $2(2.3 \%)$ & $174(98.9 \%)$ & $2(1.1 \%)$ \\
\hline Uighur & $62(93.9 \%)$ & $4(6.1 \%)$ & $128(97.0 \%)$ & $4(3.0 \%)$ \\
\hline Fisher & \multicolumn{2}{|c|}{1.445} & \multicolumn{2}{|c|}{1.410} \\
\hline $\mathrm{P}$ & \multicolumn{2}{|c|}{0.403} & \multicolumn{2}{|c|}{0.408} \\
\hline
\end{tabular}

\section{DISCUSSION}

Epidemiological studies have shown that some Japanese families with high-HDL hyperlipidemia have high frequencies of the mutation $\mathrm{D} 442 \mathrm{G}$ in intron 14 of the CETP gene, and the frequency of the GA mutation in exon 15 was also high. These mutations cause changes in the quantity, shape, and function of HDL and LDL. The R451Q mutation is found only in Caucasian populations in Europe and the United States, ranging in frequency from 0.019 to 0.038. In a Chinese Han population study, the R451Q mutation was not detected, indicating that this mutation is very rare in Han Chinese. Rejeb et al. (2012) reported that the Q allele of the R451Q polymorphism is associated with a decrease in HDL and an increase in ApoB and risk of CHD.

The incidence of CHD in Uyghur subjects is higher, and it is important to determine the genetic basis for this increased susceptibility to CHD in this ethnic group. This study found that the G/A mutation rate in the Han and Uyghur subjects in CHD and control groups was very low, and the homozygous mutation was not detected. In addition, no difference in frequency of the heterozygous R451Q gene mutation was found between Uyghur and Han subjects. Moreover, mutation frequency and blood lipid levels were all very similar in the Uyghur and Han subjects and in the CHD, CHD combined with diabetes, and control groups. In this study, the G/A mutation frequencies in subjects of the CHD, CHD combined with diabetes, and the control group were lower than those in the subjects from the Japanese population, which was consistent with domestic reports. Although different races may carry different gene polymorphisms, this gene polymorphism was not different in the Han and Uyghur populations. The results of this study suggest that the R451Q polymorphism in the CETP gene is not one of susceptibility genes for CHD in Han Chinese and Xinjiang Uyghur. However, because of the small sample size, further studies are still needed.

The variation in the association of CETP with different population groups and with CHD and plasma lipid levels is due to different genetic backgrounds, environmental effects, and different clinical criteria. The structure, function, and metabolism of lipoproteins in the patients with CETP gene mutations, especially CETP mutations affecting metabolic regulation of HDL levels, require additional detailed studies to determine the relationship between CETP and atherosclerosis at the molecular level, to correctly evaluate the pathophysiology and clinical significance of CETP.

\section{REFERENCES}

Barter PJ, Caulfield M, Eriksson M, Grundy SM, et al. (2007). Effects of torcetrapib in patients at high risk for coronary events. N. Engl. J. Med. 357: 2109-2122.

Boekholdt SM and Thompson JF (2003). Natural genetic variation as a tool in understanding the role of CETP in lipid levels and disease. J. Lipid Res. 44: 1080-1093. 
Inazu A, Jiang XC, Haraki T, Yagi K, et al. (1994). Genetic cholesteryl ester transfer protein deficiency caused by two prevalent mutations as a major determinant of increased levels of high density lipoprotein cholesterol. J. Clin. Invest. 94: $1872-1882$.

Kakko S, Tamminen M, Kesaniemi YA and Savolainen MJ (1998). R451Q mutation in the cholesteryl ester transfer protein (CETP) gene is associated with high plasma CETP activity. Atherosclerosis 136: 233-240.

Kathiresan S, Willer CJ, Peloso GM, Demissie S, et al. (2009). Common variants at 30 loci contribute to polygenic dyslipidemia. Nat. Genet. 41: 56-65.

Khovidhunkit W, Chartyingcharoen P, Siriwong S, Limumpornpetch P, et al. (2012). Resequencing CETP, LIPC and LIPG genes in Thai subjects with hyperalphalipoproteinemia. Am. J. Cardiol. 110: 62-66.

Liu S, Schmitz C, Stampfer MJ, Sacks F, et al. (2002). A prospective study of TaqIB polymorphism in the gene coding for cholesteryl ester transfer protein and risk of myocardial infarction in middle-aged men. Atherosclerosis 161: 469-474.

Lu H, Inazu A, Moriyama Y, Higashikata T, et al. (2003). Haplotype analyses of cholesteryl ester transfer protein gene promoter: a clue to an unsolved mystery of TaqIB polymorphism. J. Mol. Med. 81: 246-255.

Maruyama T, Sakai N, Ishigami M, Hirano K, et al. (2003). Prevalence and phenotypic spectrum of cholesteryl ester transfer protein gene mutations in Japanese hyperalphalipoproteinemia. Atherosclerosis 166: 177-185.

McCaskie PA, Beilby JP, Chapman CM, Hung J, et al. (2007). Cholesteryl ester transfer protein gene haplotypes, plasma high-density lipoprotein levels and the risk of coronary heart disease. Hum. Genet. 121: 401-411.

Ordovas JM (2000). Genetic polymorphisms and activity of cholesterol ester transfer protein (CETP): should we be measuring them? Clin. Chem. Lab. Med. 38: 945-949.

Ordovas JM, Cupples LA, Corella D, Otvos JD, et al. (2000). Association of cholesteryl ester transfer protein-TaqIB polymorphism with variations in lipoprotein subclasses and coronary heart disease risk: the Framingham study. Arterioscler. Thromb. Vasc. Biol. 20: 1323-1329.

Papp AC, Pinsonneault JK, Wang D, Newman LC, et al. (2012). Cholesteryl Ester Transfer Protein (CETP) polymorphisms affect mRNA splicing, HDL levels, and sex-dependent cardiovascular risk. PLoS One 7: e31930.

Park KW, Choi JH, Kim HK, Oh S, et al. (2003). The association of cholesteryl ester transfer protein polymorphism with high-density lipoprotein cholesterol and coronary artery disease in Koreans. Clin. Genet. 63: 31-38.

Rejeb J, Omezzine A, Boumaiza I, Rebhi L, et al. (2012). Four polymorphisms of cholesteryl ester transfer protein gene and coronary stenosis in a Tunisian population. J. Cardiovasc. Med. 13: 546-553.

Spielmann N, Leon AS, Rao DC, Rice T, et al. (2007). CETP genotypes and HDL-cholesterol phenotypes in the HERITAGE family study. Physiol. Genomics 31: 25-31.

Yamashita S, Hirano K, Sakai N and Matsuzawa Y (2000). Molecular biology and pathophysiological aspects of plasma cholesteryl ester transfer protein. Biochim. Biophys. Acta 1529: 257-275. 